\title{
A STUDY OF THE FIRE SMOKE PROPAGATION IN SUBWAY STATION UNDER THE EFFECT OF PISTON WIND
}

\author{
Wei ZHONG ${ }^{\text {a }}$, Rui TU ${ }^{\mathrm{a}}$, Jian Peng YANG ${ }^{\mathrm{b}}$, Tian Shui LIANG ${ }^{\mathrm{a}}$ \\ ${ }^{a}$ School of Chemical Engineering and Energy, Zhengzhou University, 450001 Zhengzhou, China \\ ${ }^{b}$ Fire Bureau of Henan province, Nanyang Fire Detachment, 473000 Nanyang, China
}

Received 14 Sep 2012; accepted 05 Nov 2012

\begin{abstract}
A running traffic train induces piston wind in the subway. The influence of piston wind on fire smoke propagation in subway is investigated numerically. The flow field structure in fire platform, temperature contours and velocity profiles at certain positions are obtained at various scenarios respectively. Three methods are adopted to reduce the impact of piston wind on smoke layers. Results show that large-scale vortexes and tremendous horizontal inertial force would be produced under the influence of piston wind; and that smoke stratification would be broken totally under its influence, therefore toxic gas would spread to subway hall through stairs. So the former smoke management system in a subway station becomes less effective. Results also show that combination of enhanced the volume flux of pressurization at the subway hall and lowering the height of smoke screens around stairs are necessary to restrict hazard smoke on the floor on fire. The bypass wind tunnel and ventilation shaft are useful to attenuate the magnitude of piston wind.
\end{abstract}

Keywords: subway fire, tunnel, piston wind, pressurization.

\section{Introduction}

The overall length of worldwide subway has been increasing due to traffic congestion, which is an outstanding problem for metropolises. To date, about 200 cities worldwide have built their own subway/light rail system with the full operation length exceeding 6000 kilometers (Cao 2003). It is common knowledge that most fire deaths result from smoke and toxic gas. Fires in subways are more dangerous as stations are enclosed spaces located under the ground, usually full of passengers. Consequently, fire in a subway station can result in disastrous casualties. In 1995, the Baku Metro fire caused 558 deaths and 269 injuries (Chen et al. 2003). The Daegu subway fire resulted in 198 deaths and 146 injuries in 2003. According to statistics, $85 \%$ of building fire victims died from toxic smoke (Brennan 1999). Therefore, the design of smoke control systems is very important to ensure personal safety. And, the effect of smoke control system may be influenced by many other factors (Li et al. 2010a, b), such as the water droplets sprayed by sprinklers, the exterior wind, etc.

The connection between tunnels and platform of a subway station has three types: closed, semi-closed and open. So subway stations can be classified into three types respectively. The open type station was applied in subway system at early stage, and is often used for some high latitude countries currently. When train runs in a tunnel, the air movement is driven along the tunnel and air flow through the tunnel walls is largely suppressed. This phenomenon is called Piston Effect (Chen et al. 1998), and the airflow caused by running train is called piston wind. For the semi-enclosed type or open type subway stations, the piston wind would directly affect the propagation of fire smoke. The stratification of smoke layers would be broke by horizontal inertial force (Li et al. 2010a, b) which is relevant to the piston wind. When a fire occurs in platform of subway stations, two emergency managements of vehicles are often used in China. One is stopping vehicle in tunnel and evacuating passengers. The other is passing the station on fire and parking in the next station. Thus, the smoke propagation in subway stations would be effected by the piston wind greatly in the second situation.

On the subway fire, a lot of research work has been conducted in recent years. Chen et al. (2003) investigated the three-dimensional smoke flow fields under various fires scenarios in a representative subway station of Taipei Rapid Transit System, and clarified the mechanisms of smoke competition at stairs deduced to the stack effect. Ji et al. (2011) studied theoretically and experimentally the maximum smoke temperature under the ceiling and proposed a correlation determining the maximum smoke temperature by taking the end wall effect into account. Moriyama et al. (2005) discussed the experimental

Corresponding author: Tian Shui Liang

E-mail: liangtsh@zzu.edu.cn 
and numerical studies on smoke movement and control in subway stations. The experiments were conducted in three actual stations in service using small methanol pool fires as the fuel to generate data basis to validate and tune numerical models for subway stations fires and furthermore to examine the performance of smoke control systems. Zhang et al. (2008) conducted a numerical simulation of kerosene fire/smoke spread over the subway stations platform by the software CFD and SIMPLE algorithm for fluid mechanics, including five different types of smoke exhaust systems. The results showed that when the mechanical smoke exhauster is used solely, the position difference of exhauster outlets affects unobviously the fire/smoke control effectiveness. But, the exhauster in combination with forced draft system can inhibit more efficiently the spread of fire/smoke though periodical attenuation is found during the inhibition. Gao et al. (2012) studied the hybrid ventilation with the dispersion of fire-induced buoyancy driven smoke in a subway station. Heat release rate, smoke temperature in the vertical direction, smoke layers height and $\mathrm{CO}$ concentration were validated with experimental data, and good agreement is achieved. The effect of hybrid ventilation on $\mathrm{CO}$ concentration reduction and its suppression effect on horizontal dispersion of smoke are discussed.

Juraeva et al. (2011) focuses on computational analysis for the improvement of the tunnel ventilation and the environment control system of the subway by solving two-equation turbulence model for train-induced unsteady flow. Ricco et al. (2007) studied the pressure waves generated by a train experimental and numerically to understand the flow field in a standard configuration tunnel with airshafts. Li (2005) conducted a series of tests in Beijing underground railway system, summarizes the velocity profiles of piston wind in tunnel, and studied the influence of piston wind on the metro environment. Zhang (2007) tested the velocity and temperature of piston wind under different conditions in a subway station of Tianjin, the result shown that the bypass wind tunnels cut down the volume of air flowing into the station and reduce the effect of subway stations thermal environment. At the same time, a CFD model was built based on the practical subway stations, which was used to simulate the velocity field and temperature field by software Airpak, and make a validation with tests. J. Y. Kim and K. Y. Kim (2007) analysed the unsteady three-dimensional flow in the subway tunnel caused by a train movement, presented the pressure and air velocity variations with time by using software CFX4, and made a confirmation by a $1 / 20$ scale model.

Researches on influence of piston wind on flow structure in subway stations are relatively little, and most of these works focus on the environmental control under the function of piston wind. The smoke propagation under the effect of piston wind has not been reported. A 3D geometric model is established based on a real subway station; a commercial fluid dynamic software FLUENT is adopt as the solver. The paper study the interaction of piston wind with fire smoke in platform layer, and propose valid methods to optimize smoke manage system of subway stations.

\section{Physical model}

The subway station is consist of a platform layer, a subway hall layer and two tunnels adjacent to platform, each layer is $130 \mathrm{~m}$ long, $16 \mathrm{~m}$ wide and $4 \mathrm{~m}$ high, as shown in Figure 1. There are four exits locate in the four corners of subway hall; the size of the exits is $3 \times 4 \times 2 \mathrm{~m}$. The platform is an island style, and there are no screen door between platform and tunnels. The platform is divided into four smoke bays by the smoke screens beneath the ceiling. The platform layer is connected with hall layer by four stairs, which numbered with $1,2,3$, and 4 . The area of stair's cross section is $15 \mathrm{~m}^{2}$, the orientation of number 4 is contrary to others, and there are smoke screens around four stairs. The smoke exhaust vents are set beneath the ceiling of platform, each vent's area is $0.5 \mathrm{~m}^{2}$, and is $3 \mathrm{~m}$ high from the ground of platform. The air inlets are set in the both side of hall layer beneath ceiling, the volume flux of pressurization can be adjusted according to the fire size.

The section of tunnels on the both side of platform is simplified to a rectangle with $4 \mathrm{~m}$ wide and $5 \mathrm{~m}$ high, the north tunnel is set to $230 \mathrm{~m}$ long because there has no train run in it, and the south tunnel is set to $2200 \mathrm{~m}$ long. The train is simplified to a hexahedron with $114 \mathrm{~m}$ long, $3 \mathrm{~m}$ wide and $4 \mathrm{~m}$ high, which is located in the entrance of the south tunnel at the beginning. Bypasses wind tunnel and vertical shafts are added in some scenarios in order to consider the influence of the bypass wind tunnel and vertical shaft to the piston wind, and the section of the bypass and shaft are $3.5 \times 3 \mathrm{~m}$ and $4 \times 6 \mathrm{~m}$ respectively.

The fire source is located in the middle of the $1^{\text {st }}$ and $2^{\text {nd }}$ stairs, the peak value of heat release rate is assumed as $2 \mathrm{MW}$ to simulate the baggage fire (Ji 2010a). When a fire occurs, the smoke extraction system in platform layer start to discharge fire smoke, and the pressurization system in hall layer begin to blow air. According to the code of China Standards (GB50157 2003), the volume flux of mechanical fan in platform is $60 \mathrm{~m}^{3} / \mathrm{m}^{2} \cdot \mathrm{h}$. In hall layer, the volume flux of pressurization system is $120000 \mathrm{~m}^{3} / \mathrm{h}$; the flux value could be enhanced according to the fire size.

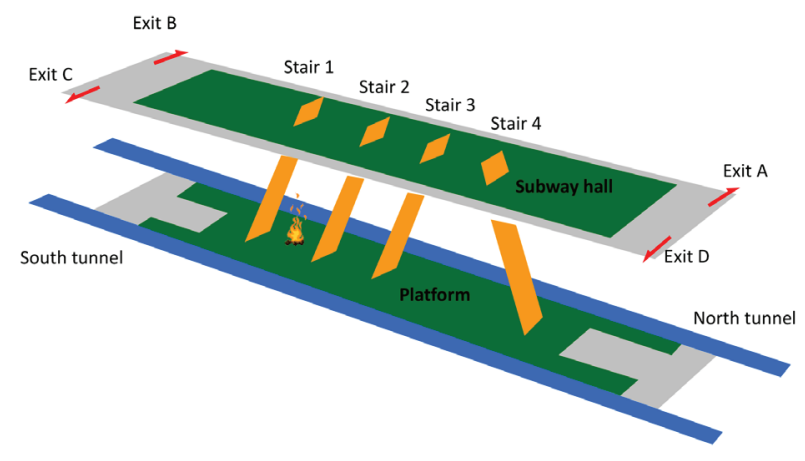

Fig. 1. The sketch map of subway station 
Flow field of platform would change complexly by the combined effect of buoyancy plumes, piston wind and smoke management system. The temperature field would change correspondingly as well. In order to monitor the change of temperature field, three groups of thermocouples are positioned vertically on the middle section of platform, as shown in Figure 2; thermocouples in one group is spaced at vertical interval of $0.25 \mathrm{~m}$.

After fire free burning for $120 \mathrm{~s}$, train would start of from the entrance of south tunnel. The train would reach its peak velocity of $20 \mathrm{~m} / \mathrm{s}$ (Yang 2007) at $130 \mathrm{~s}$, and maintains this speed, as shown in Figure 3. This mode of train running is denoted as mode 1 . As a contrast scenario, fire smoke propagation without piston wind is also solved, and this mode is denoted as mode 2 which indicate there is no train running in the tunnel.

Six scenarios are designed and investigated in this paper, as shown in Table 1. The effect of enhancing volume flux of pressurization in subway hall, lowering smoke screen around stairs and setting bypass wind tunnel and vertical shaft are verified respectively.

\section{CFD numerical modelling}

\subsection{The basic governing equation}

Based on the assumption, the platform fire and the 3D flow of piston wind can be described by the basic governing equations (Fluent Help 2009), which are shown below.

The mass conservation equation:

$$
\frac{\partial \rho}{\partial t}+\frac{\partial}{\partial x_{i}}\left(\rho u_{i}\right)=0
$$

The momentum equation:

$$
\frac{\partial}{\partial t}\left(\rho u_{i}\right)+\frac{\partial}{\partial x_{j}}\left(\rho u_{i} u_{j}\right)=-\frac{\partial p}{\partial x_{i}}+\frac{\partial \tau_{i j}}{\partial x_{j}}+\rho g_{i}+F_{i}
$$

where: $P$ is static pressure; $\tau_{i j}=\mu\left(\frac{\partial u_{i}}{\partial x_{j}}+\frac{\partial u_{j}}{\partial x_{i}}-\frac{2}{3} \delta_{i j} \frac{\partial u_{i}}{\partial x_{i}}\right)$

is shear stress tensor, $\mu$ is viscosity; $r g_{i}$ is the body force in the direction of $i ; F_{i}$ is the source.

The energy equation:

$$
\frac{\partial}{\partial t}(\rho h)+\frac{\partial}{\partial x_{i}}\left(\rho u_{i} h\right)=-\frac{\partial}{\partial x_{i}}\left(k+k_{i}\right) \frac{\partial T}{\partial x_{i}}+S_{h}
$$

where: $k$ is the thermal conductivity of molecule; $K i=$ $C_{p} \mu / \operatorname{Pr}$ is the thermal conductivity caused by turbulence

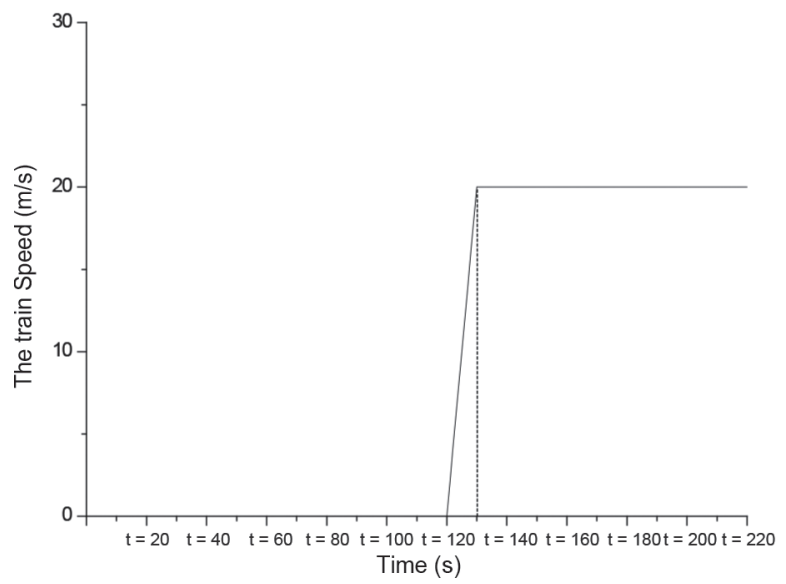

Fig. 3. The running chart of train

Table 1. Fire scenarios list

\begin{tabular}{lccccc}
\hline & $\begin{array}{c}\text { Distance } \\
\text { from } \\
\text { bottom } \\
\text { retaining } \\
\text { wall of } \\
\text { smoke s } \\
\text { to floor } \\
(\mathrm{m})\end{array}$ & $\begin{array}{c}\text { Volume } \\
\text { flux of } \\
\text { pressurization } \\
\left(\mathrm{m}^{3} / \mathrm{h}\right)\end{array}$ & $\begin{array}{c}\text { Bypass/ } \\
\text { shaft }\end{array}$ & $\begin{array}{c}\text { Train's } \\
\text { move } \\
\text { mode }\end{array}$ & $\begin{array}{c}\text { HRR } \\
(\mathrm{MW})\end{array}$ \\
\hline Case 1 & 3 & 0 & no & 1 & 0 \\
\hline Case 2 & 3 & 120000 & no & 2 & 2 \\
\hline Case 3 & 3 & 120000 & no & 1 & 2 \\
\hline Case 4 & 3 & 360000 & no & 1 & 2 \\
\hline Case 5 & 2.3 & 360000 & no & 1 & 2 \\
\hline Case 6 & 2.3 & 360000 & yes & 1 & 2 \\
\hline
\end{tabular}

diffusion; Pr is the Planck constant; $S_{h}$ is the volumetric heat sources.

\subsection{The turbulence model}

The $K-\varepsilon$ model has a good balance on calculated amount and calculation precision, and has been used to solve engineering turbulence widely. The two-equation model $K-\varepsilon$ is used to solve the smoke turbulent flow, and buoyancy source term is activated (Wang 2007). The governing equation of turbulent kinetic energy $k$ and the turbulent dissipation $\varepsilon$ is shown below:

$$
\rho \frac{\partial k}{\partial t}=\frac{\partial}{\partial x_{j}}\left[\left(\mu+\frac{\mu_{t}}{\sigma_{k}}\right) \frac{\partial k}{\partial x_{j}}\right]+G_{k}+G_{b}-\rho \varepsilon ;
$$

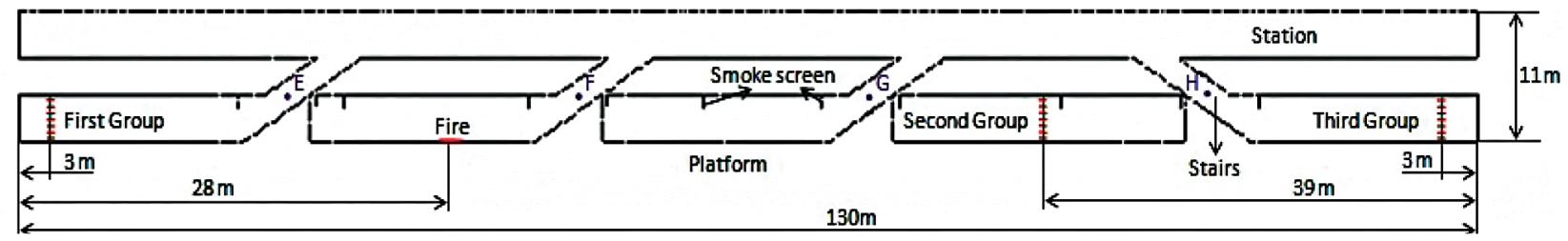

Fig. 2. The thermocouples in the middle section of platform 


$$
\begin{aligned}
& \frac{\partial(\rho \varepsilon)}{\partial t}=\frac{\partial}{\partial x_{j}}\left[\left(\frac{\mu_{t}}{\sigma_{\varepsilon}} \frac{\partial \varepsilon}{\partial x_{j}}\right) \frac{\partial k}{\partial x_{j}}\right]+\frac{\varepsilon}{k} ; \\
& {\left[c_{1}\left(G_{k}+G_{b}\right)\left(1+c_{3} R_{f}\right)\right]-c_{2} \rho \varepsilon,}
\end{aligned}
$$

where $\mu t=C_{\mu} r k^{2} / \varepsilon, C_{\mu}=0.09 ; G_{k}, G_{b}$ is the turbulent kinetic energy production term generated by velocity gradient and buoyancy respectively, the constant $C_{1}=1.44$, $C_{2}=1.92, C_{3}=0.8, S_{k}=1.0, S_{\varepsilon}=1.3$.

\subsection{The dynamic mesh model}

To get grid independent solution, the grid sizes change from $0.5 \mathrm{~m}$ to $0.3 \mathrm{~m}$ by $0.1 \mathrm{~m}$. The grid size is $0.3(\mathrm{x}) \times 0.3(\mathrm{y}) \times 0.3(\mathrm{z}) \mathrm{m}$ for tunnel and is $0.4(\mathrm{z}) \times 0.4(\mathrm{x}) \times$ $0.4(\mathrm{y}) \mathrm{m}$ for the others region.

To model the moving geometry of train, the dynamic mesh model is adopted. In Fluent 6.3.26 (2009), three groups of mesh motion methods are available to update the volume mesh in the deforming regions subject to the motion defined at the boundaries: smoothing methods, dynamic layering and local re-meshing methods (Zhang et al. 2010). Because of the characteristics of motion of train in subway tunnel, the dynamic layering method is chosen in this study. With the dynamic layering method, layers of cells adjacent to moving boundaries can be created or removed based on the size of the layer adjacent to the moving boundaries. With the motion of train, the volume meshes is updated by the Fluent at each time step. During iterative processes, the residual of every equation is monitored. The convergence criterion is $10^{-5}$ for all variables except the energy is $10^{-6}$.

\subsection{Boundary condition}

The four exits of subway hall and four tunnels entrances are set as pressure-outlet boundary condition. Smoke vents in platform and pressurization air inlets in subway hall are set as velocity-inlet boundary condition. The initial pressure and ambient pressure is 1 ATM and the initial temperature and ambient temperature is $298 \mathrm{~K}$. Pressure-based SIMPLIC algorithm is used to solve the pressure-velocity coupling (Kim, J. Y., Kim, K. Y. 2009).

\section{Results and discussion}

\subsection{Flow field in platform under the effect of piston wind}

In order to analyse the influence of piston wind to the flow structure of platform, velocity data are extracted at eight positions respectively marked as $\mathrm{A}$ to $\mathrm{H}$ between tunnels and platform, as well as at the intermediate positions of four stairs, as shown in Figure 4. Velocity variation at the eight points obtained through simulation under no fire circumstances is shown in Figures 5 and 6. The velocity direction is defined as that positive value means gas flowing into platform while negative value means flowing out.

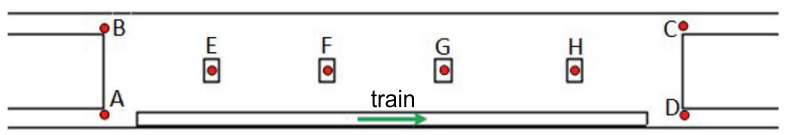

Fig. 4. The sketch of velocity measuring points on section $\mathrm{Z}=1.7 \mathrm{~m}$ in case 1

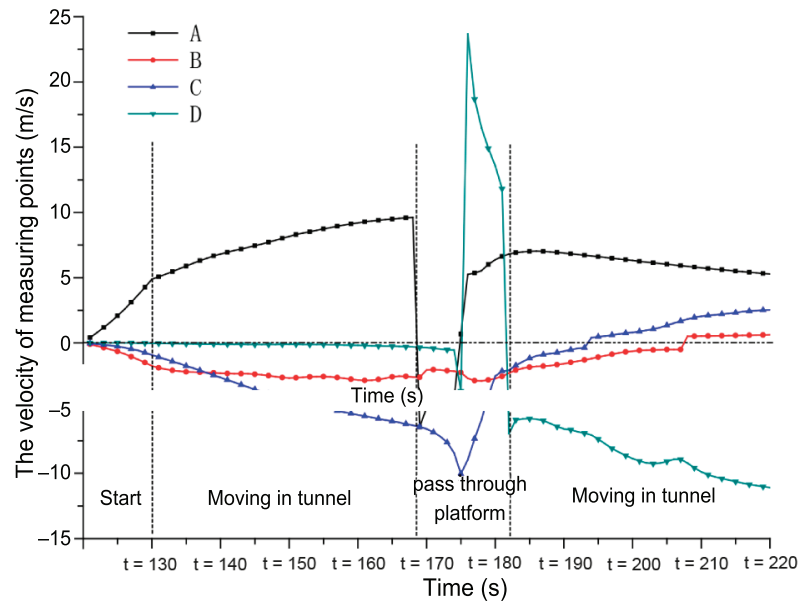

Fig. 5. The velocity variation at four entrance of tunnels in case 1

Figure 5 shows the velocity variation with time at tunnel entrances on both sides of the platform. The running of vehicle is divided into four phases, where the first one is up to $130 \mathrm{~s}$ during which period the vehicle gets accelerated from still to $20 \mathrm{~m} / \mathrm{s}$; the second is up to $168 \mathrm{~s}$ during which period the vehicle enters the tunnel till its head moves out of the tunnel. In the first two phases, the main factor resulting in the piston wind's effect on the flow field in platform is the positive pressure area near the vehicle head. The third phase is up to $182 \mathrm{~s}$ during which period the vehicle runs by the platform, its tail moves out of the tunnel at $175 \mathrm{~s}$ and its head arrives at the right tunnel meanwhile, and then at $182 \mathrm{~s}$ the whole vehicle gets inside the tunnel. In this phase, the flow filed structure is subject to influence from the high pressure area near the head, the low pressure area behind the tail, and the main structure itself when the whole vehicle runs aside the platform. In the last phase, the whole vehicle runs inside the tunnel, during this time the aforesaid influence is mainly a result of the negative pressure area behind the tail.

It can be seen from Figure 5 that the velocity at point $\mathrm{A}$ is positive and increases during the first two phases. This is because piston wind is gradually formed as the vehicle moving to the platform. The velocity keeps increasing before the whole vehicle moving out of the tunnel when the high pressure area near the head gets closer to the platform. Since point A is just at the intermediate part relative to the vehicle when it's running out from the tunnel, the velocity at this point turns to be negative immediately since part of air flow in front of the head flows through it towards the tail. The air flow 
behind the tail would follow the vehicle driven by pressure difference due to the low pressure as the tail passing point A. Therefore, the velocity at Point A turns to be positive again. However, it would decrease gradually as the vehicle running further from the platform.

The velocity at point $B$ keeps negative at about $-2 \mathrm{~m} / \mathrm{s}$ before the vehicle completely gets inside the platform. This means that the air flows through this point towards the tunnel most of time when the vehicle is running and a minor part of air gets through it towards the platform when the vehicle moves far away from the station. The velocity at point $C$ keeps increasing in value and reaches its peak value of $10 \mathrm{~m} / \mathrm{s}$ before the vehicle starts to enter the tunnel again. This is because point $\mathrm{C}$ gets closer with the high pressure area near the head as the vehicle running. We can also determine that point $\mathrm{C}$ is an important channel for the piston wind to flow out of the platform. The velocity at this point would decrease gradually due to the sharp drop of pressure difference between this point and the vehicle as the head enters the tunnel again. However, the velocity here turns to be positive under chimney effect when the whole vehicle enters the runnel, leaving the air flows from the tunnel to the platform.

The velocity at point $\mathrm{D}$ is very low at the beginning and turns to increase rapidly when the vehicle arrives at this point because of air flow around from the high pressure area near the head to the low pressure area behind the tail. When the whole vehicle enters the tunnel, a lot of air is sucked into the tunnel towards the low pressure area, leaving the velocity at this point turns to be negative moving from the platform to the tunnel.

Figure 6 represents wind velocity variation at four stair outlets during running of the vehicle. As shown in this figure, the wind at four stairs flows towards the hall from the platform before $140 \mathrm{~s}$, when the vehicle has a further distance from the platform, so the piston wind from point $\mathrm{A}$ to the platform is slower, and it has the maximum influence on stair 1 which is the closest to point $\mathrm{A}$, then the wind velocity at outlet of stair 1 reaches the peak value of $4 \mathrm{~m} / \mathrm{s}$ when it is up to $140 \mathrm{~s}$. Afterward,

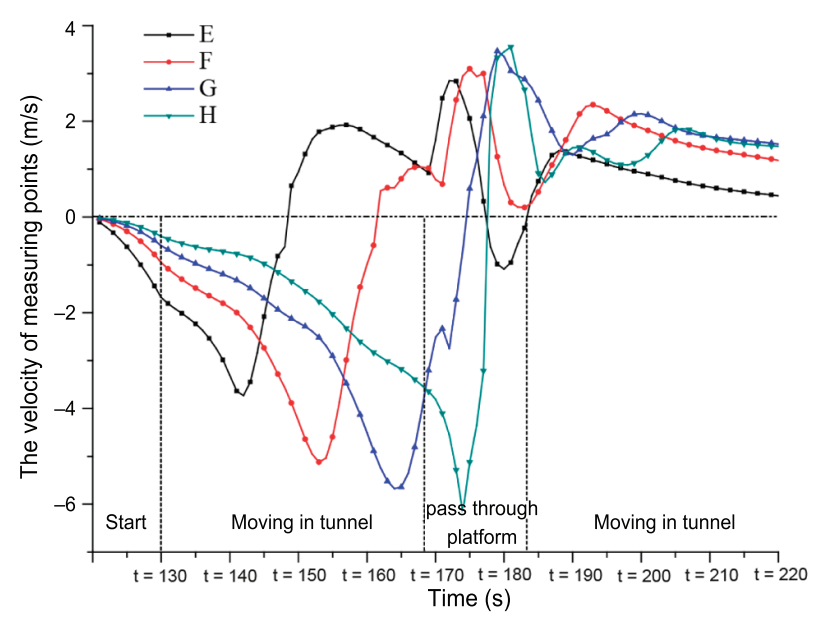

Fig. 6. The velocityvariationof four stairs in case 1 with the increase of the piston wind, the area which is influenced maximally by the piston wind moves towards stair 2 gradually, whereas the wind velocity falls little by little. When it is up to $150 \mathrm{~s}$, a plenty of air enters the hall through stair 2 so that the hall pressure near stair 2 exceeds that at stair 1 , at this time, part of air flowing into the hall flows back to the platform, and the wind velocity reverses at the outlet of stair 1. Similarly, with the running of the vehicle, the piston wind velocity is getting higher and higher, the area which is influenced maximally by the piston wind moves forward constantly, the velocity at the subsequent stair outlets reaches the peak value successively and the backflow occurs subsequently at the outlets of stair 2 and 3 . when it is up to $177 \mathrm{~s}$, the vehicle head enters the tunnel on the right side, meanwhile the vehicle tail runs out of the tunnel on the left side, the air in the metro tunnel flows into the platform under low pressure area at the vehicle tail, which generates the airflow up to $5 \mathrm{~m} / \mathrm{s}$ and causes another variation of the flow direction at stair 1 , when the vehicle tail runs through stair 2, 3 and 4, the air velocity from the vehicle tail falls because the platform is too broad, therefore, the velocity curve only falls but not reverses. After the vehicle keeps away from the platform and enters the tunnel on the right side, the wind velocity at four stair outlets starts to fall successively.

Figure 7 shows the middle-section streamlines of the underground station at different moments. As a result, it shows the airflow in the stairs moves towards the hall when it is up to $135 \mathrm{~s}$, however, when it is up to $150 \mathrm{~s}$, the airflow direction at the outlet of stair 1 changes and the air flows from the hall to the platform because the air mainly flows into the hall through stair 2 . The vehicle tail reaches the middle of the platform when it is up to $180 \mathrm{~s}$, at that moment, the airflow moves towards the platform from stair 2, 3 and 4, but it reverses for the second time at the outlet of stair 1 and flows from the platform to the hall. When it is up to $210 \mathrm{~s}$, the airflow at the stair outlets moves towards the platform under suction effect.

The streamlines of the platform's flow filed on section $\mathrm{Z}=1.7 \mathrm{~m}$ at different time are shown in Figure 8. It can be seen that there are two anticlockwise vortexes nearby the stair 1 at $150 \mathrm{~s}$. This is because the sudden segregation of boundary layer would happen at the perpendicular joint between the tunnel and platform at the time when piston wind getting inside the platform through the tunnel with certain velocity. The air flow thus moving out of the tunnel would run by longitudinal direction of platform instead of the left wall of it, and a low pressure area was formed on the left platform. A part of air flows out through surface B as driven by pressure difference and forms two anticlockwise vortexes around the stair 1 .

The piston wind gets accelerated with the moving of the vehicle and reaches $10 \mathrm{~m} / \mathrm{s}$ at $165 \mathrm{~s}$ when the vehicle is about to move out of the tunnel. Therefore, the low pressure area due to segregation of section area is enlarged. 


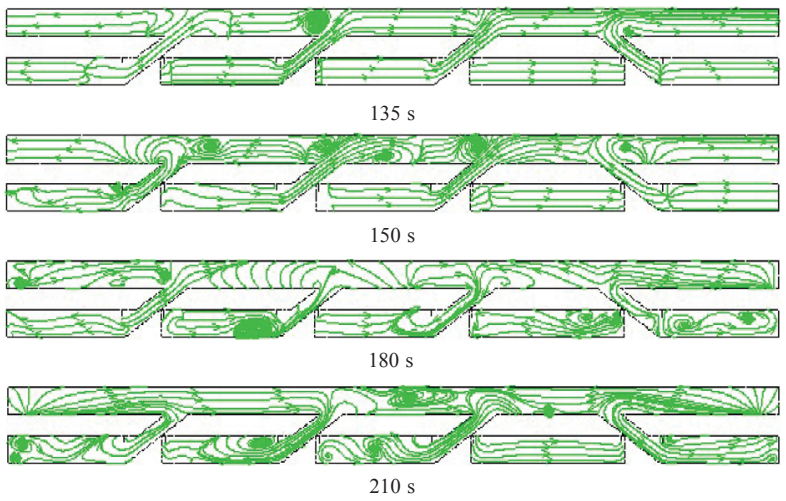

Fig. 7. The streamlines of middle-section of the station at different time
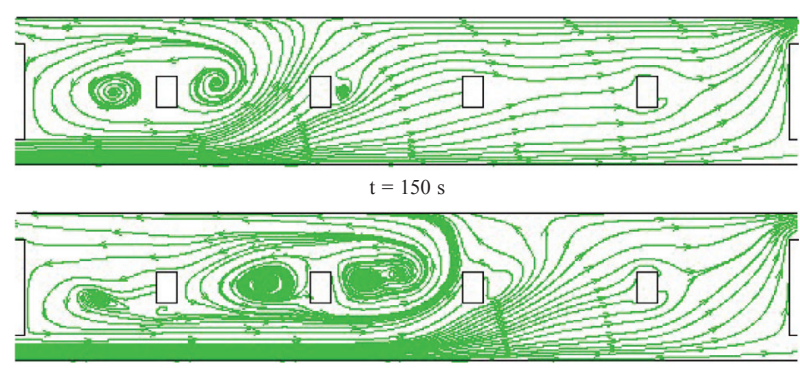

$\mathrm{t}=165 \mathrm{~s}$

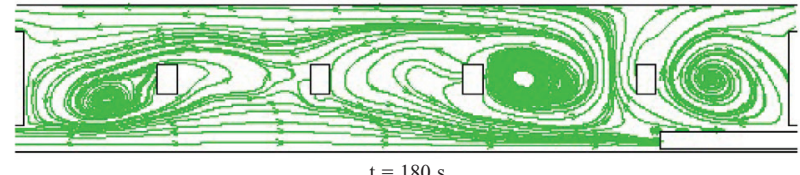

$\mathrm{t}=180 \mathrm{~s}$
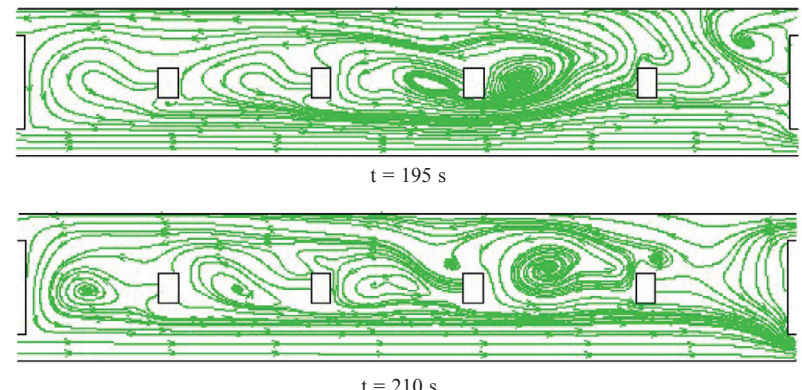

$t=210 s$

Fig. 8. Thes treamlines of section $\mathrm{Z}=1.7 \mathrm{~m}$ at different time

Stair 3 becomes the region which subject to the effect of piston wind most. When the tail gets nearby the stair 4 at $180 \mathrm{~s}$ when most part of the vehicle has entered the tunnel, three eddies would form by two air flows moving from the head to the tail and from the platform to the tail due to existence of a negative pressure area around the tail. This eddies and the piston wind's effect on the flow field is trend to disappear with the leaving of the vehicle.

According to above results, the flow field experienced complicated changes under effect of piston wind. With the movement of the train, the piston wind would lead to complex vortexes at the platform and get backward for many times. Therefore, the propagation of fire smoke would be influenced by the piston wind greatly when the platform catches a fire.

\subsection{Smoke propagation under the effect of piston wind}

Case 2 (Table 1) is a contrast scenario. The temperature contours on the middle section of platform at some moment are shown in Figure 9. Figure 9 shows that a lot of smoke is driven into subway hall at two minutes after the start of fire; that smoke would descend to floor at the left end of platform with the continuance of fire; that smoke layers height would reduce gradually with more smoke enter subway hall; that most areas of platform or subway hall are full with fire smoke at $210 \mathrm{~s}$; and that smoke stratification is still distinct at this situation.

Smoke propagation under the effect piston wind is simulated (case 3). Figure 9 presents temperature contours on middle section of platform in case 3. Compared with Figure 9, Figure 10 shows that fire plume in platform is deflective and some smoke is driven into hall layer through stair 3 at $150 \mathrm{~s}$, that the value of smoke layers height is lower than the value in case 2 . At $180 \mathrm{~s}$ when train enter tunnel again, the subway station is filled with fire smoke, and the stratification of smoke layers is totally broke as well. Because the reason is that the tremendous horizontal inertial force produced by piston wind combined with more fresh air driven into platform under the effect of piston wind, would enhance the mixture of smoke and air, and significantly decrease temperature of smoke layers.

The temperature of three groups of thermocouples in case 2 and 3 are presented in Figure 11. It can be seen that the curves are smooth and temperature decreased along platform's height in case 2, this indicated that the smoke flow steadily and the stratification in subway stations is distinctly. However, when the train enter platform (case 3), the stratification of smoke disappear immediately. All temperature profiles changed greatly and the value of all measuring points are very close. This is due to the intense mixture of fire smoke and fresh air and descending of fire smoke to floor under the impact of piston wind. From the comparison of these two cases we can see that the piston wind is very harmful to smoke control in a subway station fire.

\subsection{Enhancing the volume flux of pressurization}

The basic principle of smoke control in subway stations is restricting smoke in the fire floor. However, case 3 indicate that a lot of smoke is pushed into subway hall under the function of piston wind, which is dangerous to the occupants in subway hall. In case 4, the volume flux of pressurization in subway hall is increased to improve the effect of smoke control. The temperature contours on middle section of platform are shown in Figure 12.

Smoke propagation is restrained under the lager volume flux of pressurization. No smoke enter subway hall at $120 \mathrm{~s}$ in case 4 , on the contrary, a lot of smoke spill to subway hall through stair 2 at $120 \mathrm{~s}$ in case 3 . It is not until $150 \mathrm{~s}$ when vehicle move to the middle of left tunnel that smoke break the obstruction of the pressurization and flow into subway hall through stair 2 under the combination of 


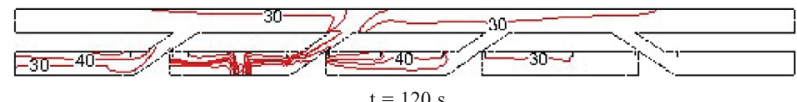

$\mathrm{t}=120 \mathrm{~s}$

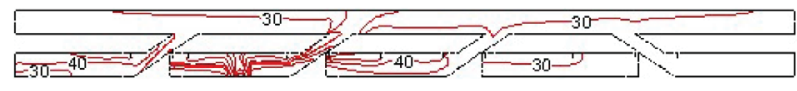

$\mathrm{t}=150 \mathrm{~s}$

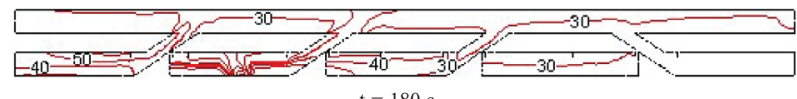

$\mathrm{t}=180 \mathrm{~s}$

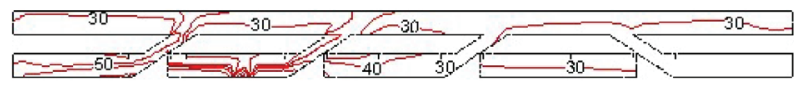

$\mathrm{t}=210 \mathrm{~s}$

Fig. 9. The temperature contours on middle section of platform in case $2\left({ }^{\circ} \mathrm{C}\right)$
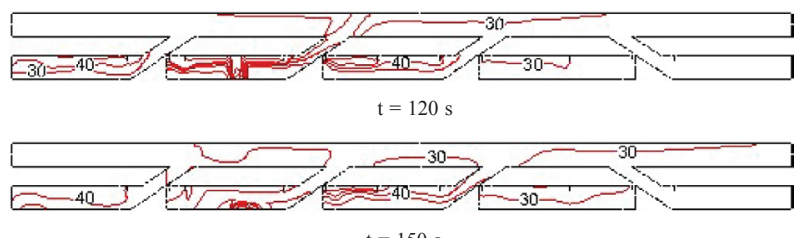

$\mathrm{t}=150 \mathrm{~s}$

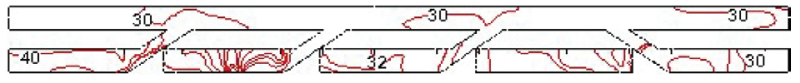

$\mathrm{t}=180 \mathrm{~s}$

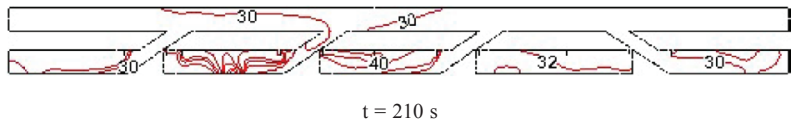

Fig. 10. The temperature contours on middle section of platform in case $3\left({ }^{\circ} \mathrm{C}\right)$

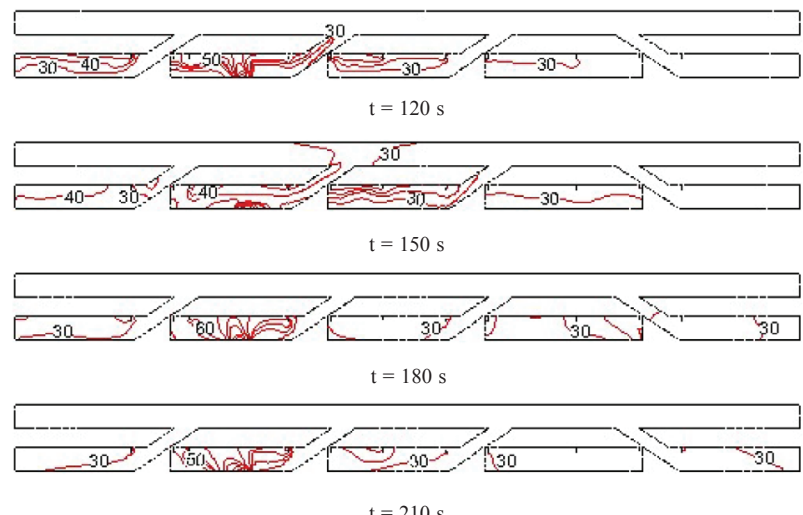

Fig. 12. The temperature contours on middle section of platform in case 4

buoyancy and piston wind. The time-consuming for smoke entering subway hall in case $4(150 \mathrm{~s})$ is later than it in case 3 (120 s), which is because the higher downward airflow at stairs is more effective to restrict smoke within fire floor. After the vehicle passed platform, smoke entering subway hall earlier would flow back into platform again, then there is no smoke in subway hall layer.

The velocity curves at monitoring position in four stairs (case 3 and 4) are presented in Figure 13. It can be seen that the airflow velocities of each stair in case 4 exceed $1.5 \mathrm{~m} / \mathrm{s}$ at the initial stage, while the critical velocity of $1.5 \mathrm{~m} / \mathrm{s}$ at stairs was prescribed in China's code. The velocity of stair 2 shows that the time for smoke starting to enter hall layer is delayed about $100 \mathrm{~s}$; and that the peak velocity of upward smoke flow at four stairs in
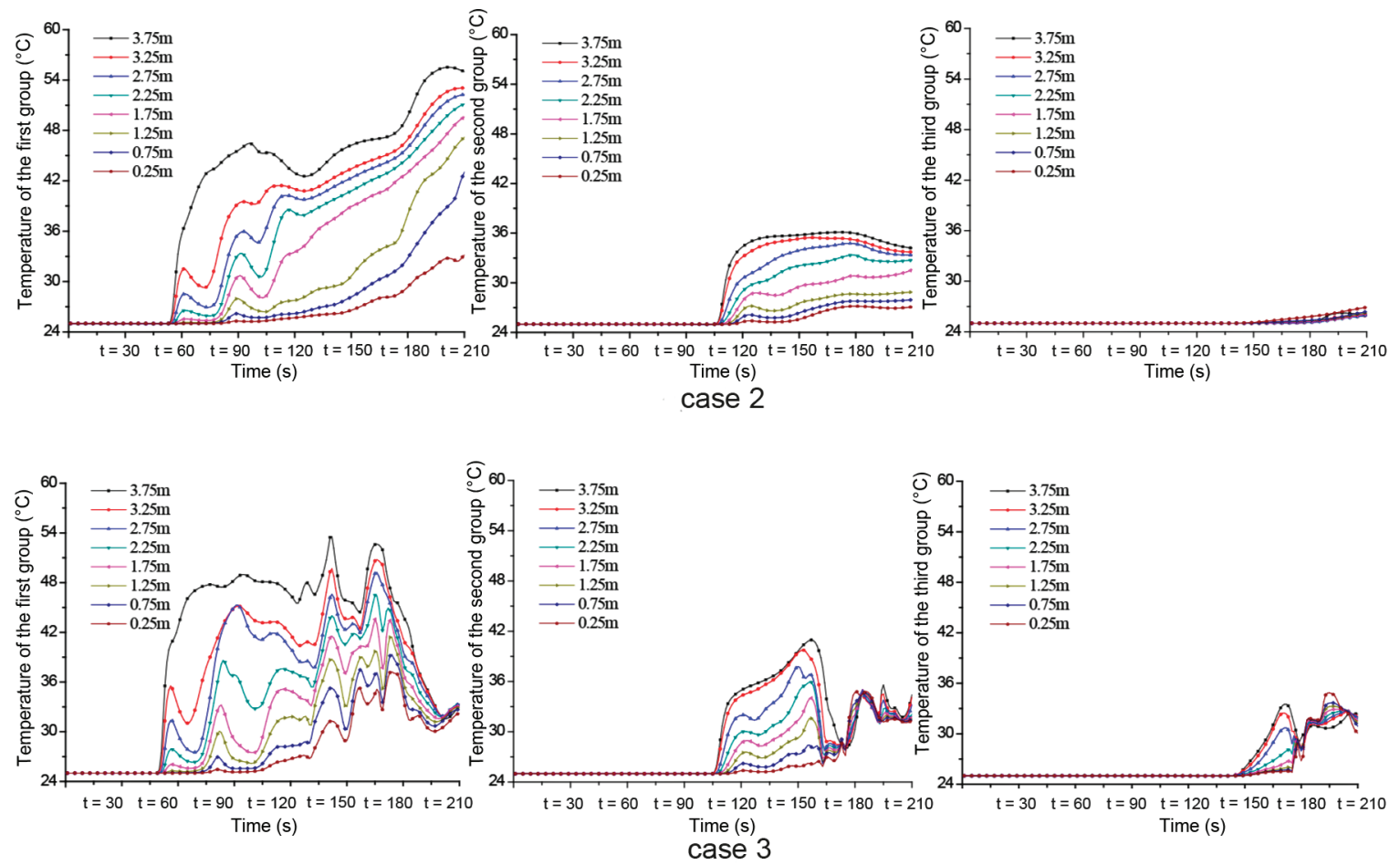

Fig. 11. The temperature variation of three groups of thermocouples in case 2 and case 3 
case 4 are less than that in case 3 . So we can conclude that the effect of smoke control system would be more effective if increasing its volume flux of pressurization.

\subsection{Increasing vertical height of retaining wall of smoke}

Enhancing the volume flux of pressurization is proved to be an effective method, but increase of volume flux of pressurization is limited in engineering practice. Ji et al. (2010b) experimentally investigated the influence of smoke vent height and exhausting velocity on mechanical smoke exhausting efficiency and found that the smoke exhausting process becomes more efficient due to the increased smoke vent height and the decreased exhausting velocity, which eliminate the plug-holing issue with fewer disturbances on the smoke layer interface. In case 5, the vertical height of retaining wall around four stairs is increased to improve the efficacy of smoke control system. According to China's code 'code for design of metro', the distance from bottom of retaining wall of smoke to the ground (around stair) can be lower to $2.3 \mathrm{~m}$. Temperature contours on middle section of platform in case 5 are shown in Figure 14. Figure 14 shows that smoke propagation is restricted by the smoke reservoir below the ceiling in case 5 . Smoke still cannot enter stair 2 at $120 \mathrm{~s}$. Smoke start to occupy stair 2 at $30 \mathrm{~s}$, but it is finally restrained in platform layer under the function of piston wind. The temperature distribution at $180 \mathrm{~s}$ and $210 \mathrm{~s}$ in case 5 are similar to the corresponding results of case 4; the temperature distribution indicates that no smoke can flow into subway hall at the whole process.

\subsection{Adding bypass tunnel and shaft}

The bypass wind tunnel and ventilation shaft are added (case 6) to weaken the effect of piston wind. Two bypass wind tunnels are added in the each side of platform, and four ventilation shafts are set between bypass tunnel and platform. The velocity of the entrance of tunnels at the four measuring points is shown in Figure 15. The temperature contours on middle section of platform in case 6 are shown in Figure 15. It can

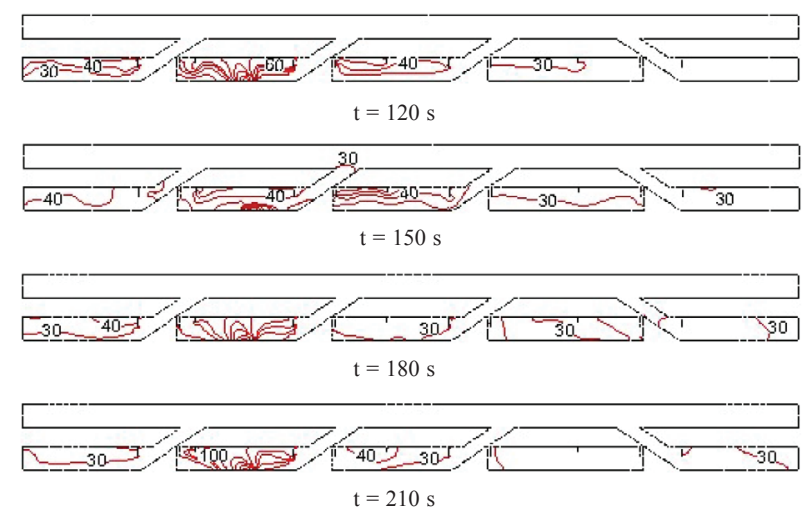

Fig. 14. The temperature contours on middle section of platform in case 5

be seen that the change temperature distribution is not obvious before the vehicle start to move. The stair 2 still not be occupied by fire smoke at $120 \mathrm{~s}$, and the right side of platform is still close to the ambient temperature at $150 \mathrm{~s}$. From the comparison of Figure 14 and Figure 16, we can see that smoke movement in platform is obviously delayed by case 6 . The reason is that piston wind is weakened by the bypass wind tunnel and ventilation shaft. The temperature distributions were very similar at $180 \mathrm{~s}$ and $210 \mathrm{~s}$ when the vehicle passed platform

The velocity at the junctions of tunnels and platform in case 5 and case 6 were illustrated in Figure 17. The results showed that the piston wind is weakened by the bypass tunnel and ventilation shafts (the peak velocity at the entrance of piston wind reduce from $10 \mathrm{~m} / \mathrm{s}$ to $7.5 \mathrm{~m} / \mathrm{s}$ ), which means the maximum volume flux of the air flowing into platform decrease by $25 \%$ before the vehicle entered platform. When the vehicle enters the right tunnel, the suction effect of vehicle is weakened similarly. The results show that air would flow into the right tunnel through the bypass tunnel; that the peak velocity at point $\mathrm{D}$ is decreased from $10 \mathrm{~m} / \mathrm{s}$ to $5 \mathrm{~m} / \mathrm{s}$; and that the direction of air flow at point $\mathrm{B}$ and $\mathrm{C}$ are not reversed in case 6 . These results

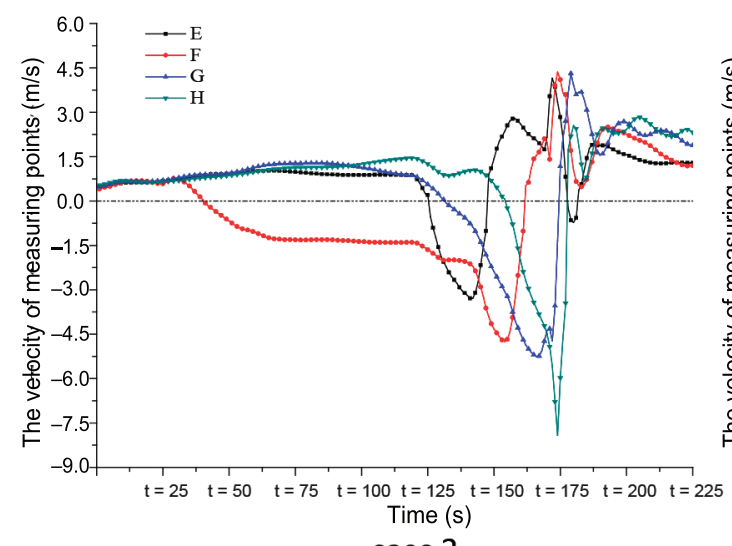

case 3

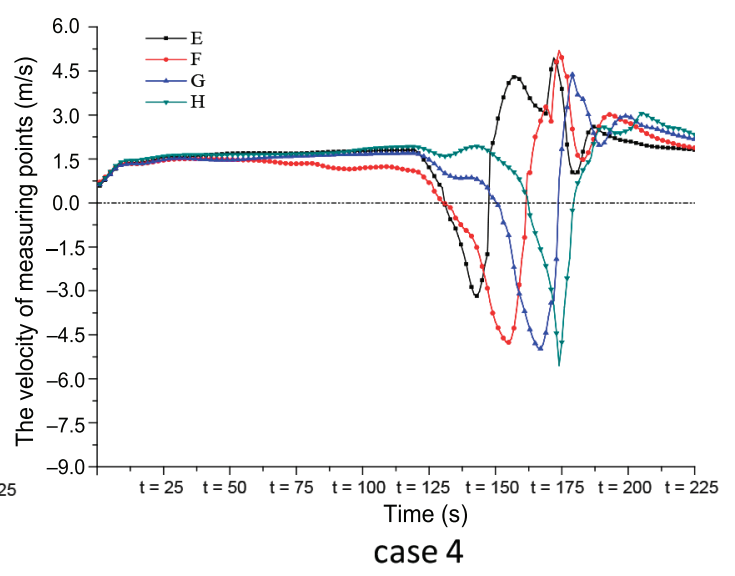

Fig. 13. The velocity at four stairs in case 3 and case 4 


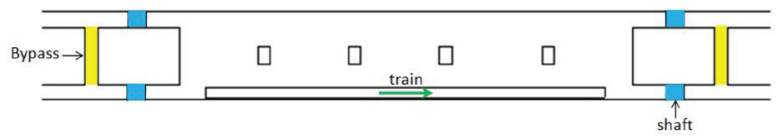

Fig. 15. The sketch map of the bypass wind tunnel and ventilation shaft

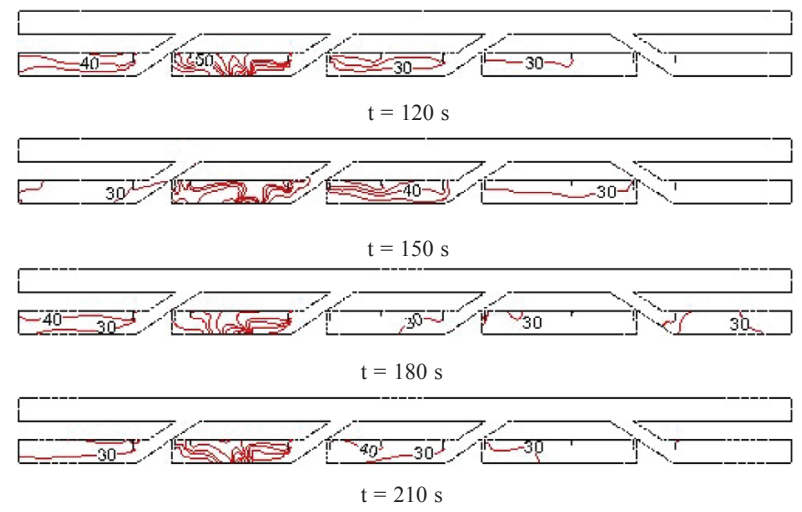

Fig. 16. The temperature contours on middle section of platform in case 6

indicate that the bypass tunnel and ventilation shaft can reduce the intensity of piston wind, decrease the peak velocity at the entrance of tunnel distinctly, and maintain the direction of four measuring points when vehicle leave platform.

\section{Conclusions}

The influence of piston wind induced by running train on smoke propagation in subway stations was investigated in this paper. Conclusions are drawn as follows.

Flow field in platform changes complicatedly under the effect of piston wind. With the entrance of running vehicle, the structure of flow field in platform is disordered and the direction of air flow at stairs and entrances of tunnels reverse several times. There are some vortex areas exist in platform, and the vortex move with the progress of vehicle. The smoke's propagation in subway stations is accelerated by the piston wind, a lot of smoke would be driven in subway hall and sucked into platform again, the mixture of smoke and air is very intense, and the stratification in subway stations is destroyed when the vehicle entered platform.

The critical velocity of downward air flow at stairs prescribed in China's code is necessary to prevent smoke flow into subway hall. Enhancing pressurization can reduce the amount of smoke which spill to subway hall effectively. The accumulation function of reservoir below the ceiling is improved by increasing vertical height of retaining wall of smoke, the combination of enhancing pressurization and increasing vertical height of retaining wall is proved to be an effective method to restrict smoke in fire floor. Adding bypass wind tunnel and ventilation shaft in tunnel can weaken the piston wind. The peak velocity at the entrance of tunnels is lowered obviously. The air flow at the junctions of tunnels and platform are not reversed when vehicle leave platform.

\section{Acknowledgements}

This work was supported by National Natural Science Foundation of China (NSFC) under Grant No. 51104132, the key technologies research and development program of Henan province under Grant No. 102102210379, and the Opening Fund of State Key Laboratory of Fire Science of University of Science and Technology of China under Grant No. HZ2011-KF07.

\section{References}

Brennan, P. 1999. Victims and survivors in fatal residential building fires, Fire and Materials 23(6): 305-310. http://dx.doi.org/10.1002/(SICI)1099-1018(199911/12) 23:6<305::AID-FAM703>3.0.CO;2-B

Chen, F. L.; Guo, S. C., et al. 2003. Smoke control of fires in subway stations, Theoretical and Computational Fluid Dynamics 16(5): 349-368. http://dx.doi.org/10.1007/s00162-002-0086-5

Chen, T. Y.; Lee, Y. T.; Hsu, C. C. 1998. Investigations of piston-effect and jet fan-effect in model vehicle tunnels, Journal of Wind Engineering and Industrial Aerodynamics 73(2): 99-110. http://dx.doi.org/10.1016/S0167-6105(97)00281-X

China Standards. GB 50157-2003, Code for design of metro. Beijing, China. 156 p.
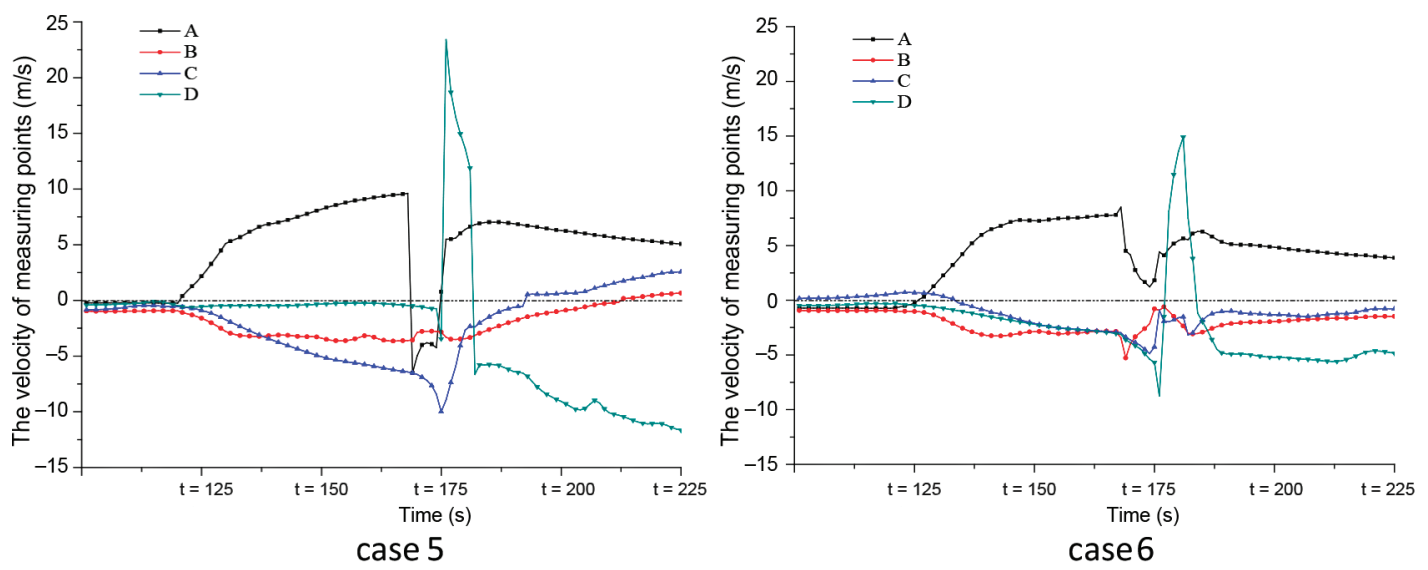

Fig. 17. The velocity at the entrance of tunnels in case 5 and case 6 
Fan, C. G.; Ji, J., et al. 2013. Experimental study of air entrainment mode with natural ventilation using shafts in road tunnel fires, International Journal of Heat and Mass Transfer 56(1-2): 750-757. http://dx.doi.org/10.1016/j.ijheatmasstransfer.2012.09.047

FLUENT Help. 2009. FLUENT 6.3 user's guide: Chapter 9. Modeling basic fluid flow, 2-4.

Gao, R.; Li, A. G., et al. 2012. Fire-induced smoke control via hybrid ventilation in a huge transit terminal subway station, Energy and Buildings 45: 280-289. http://dx.doi.org/10.1016/j.enbuild.2011.11.018

Ji, J.; Gao, Z. H., et al. 2013a. Large Eddy Simulation of stack effect on natural smoke exhausting effect in urban road tunnel fires, International Journal of Heat and Mass Transfer 66: 531-542.

http://dx.doi.org/10.1016/j.ijheatmasstransfer.2013.07.057

Ji, J.; Han, J. Y., et al. 2013b. Influence of cross-sectional area and aspect ratio of shaft on natural ventilation in urban road tunnel, International Journal of Heat and Mass Transfer 67: 420-431.

http://dx.doi.org/10.1016/j.ijheatmasstransfer.2013.08.033

$\mathrm{Ji}$, J.; Li, K. Y., et al. 2010. Experimental investigation on influence of smoke venting velocity and vent height on mechanical smoke exhaust efficiency, Journal of hazardous Materials177(1-3): 209-215.

http://dx.doi.org/10.1016/j.jhazmat.2009.12.019

Ji, J.; Zhong, W., et al. 2010. Optimization of ventilation mode during intermediate floor fire in a typical multifloor subway station, China Railway Science 31(2): $131-136$.

Ji, J.; Zhong, W., et al. 2011. A simplified calculation method on maximum smoke temperature under the ceiling in subway station fires, Tunnelling and Underground Space Technology 26(3): 490-496. http://dx.doi.org/10.1016/j.tust.2011.02.001

Juraeva, M.; Lee, J. H.; Song, D. J. 2011. A computational analysis of the train-wind to identify the best position for the air-curtain installation, Journal of Wind Engineering and Industrial Aerodynamics 99(5): 554-559. http://dx.doi.org/10.1016/j.jweia.2011.02.004

Kim, J. Y.; Kim, K. Y. 2007. Experimental and numerical analyses of train-induced unsteady tunnel flow in subway,
Tunnelling and Underground Space Technology 22(2): 166-172. http://dx.doi.org/10.1016/j.tust.2006.06.001

Kim, J. Y.; Kim, K. Y. 2009. Effects of vent shaft location on the ventilation performance in a subway tunnel, Journal of Wind Engineering and Industrial Aerodynamics 97(5-6): 174-179. http://dx.doi.org/10.1016/j.jweia.2009.06.002

Li, K. Y.; Huo, R.; Ji, J.; Ren, B. B. 2010a. Experimental investigation on drag effect of sprinkler spray to adjacent horizontal natural smoke venting, Journal of Hazardous Materials 174(1-3):512-521. http://dx.doi.org/10.1016/j.jhazmat.2009.09.082

Li, K. Y.; Spearpoint, M. J., et al. 2010b. A mathematical model on drag effect of sprinkler spray to adjacent horizontal smoke venting, Journal of Fire Protection Engineering 20(1): 27-54. http://dx.doi.org/10.1177/1042391509360270

Moriyama, S. J.; Hasemi, Y. J., et al. 2005. Smoke movement characteristics and fire safety in subway stations, Fire Science and Technology 25(3): 1462-1472.

Ricco, P.; Baron, A.; Molteni, P. 2007. Nature of pressure waves induced by a high-speed train travelling through a tunnel, Journal of Wind Engineering and Industrial Aerodynamics 95(8): 781-808. http://dx.doi.org/10.1016/j.jweia.2007.01.008

Wang, Y. F.; Zhang, J. W. 2007. CFD simulation of smoke flow and heat transfer under subway station fire, Fire Science and Technology 26(5): 485-488.

Yang, H. 2007. Numerical simulation of piston wind on platform air environment, Journal of Beijing University of Civil Engineering and Architecture 23(2): 8-13.

Zhang, L. L. 2007. The effect of bypass wind tunnel and platform bailout door on the thermal environment in subway station: Master dissertation of Tianjin University, China.

Zhang, L. P.; Deng, X. G., et al. 2010. Reviews of moving grid generation techniques and numerical methods for unsteady flow, Advances in Mechanics 40(4): 424-447.

Zhong, W.; Lv, J. J.; Li, Z. Z.; Liang, T. S. 2013. A study of bifurcation flow of fire smoke in tunnel with longitudinal ventilation, International Journal of Heat and Mass Transfer 67: 829-835. http://dx.doi.org/10.1016/j.ijheatmasstransfer.2013.08.084

Wei ZHONG. He is an Associate Professor and Vice Director of Department of Safety Science and Engineering of Zhegnzhou University. He received his PhD degree from State Key Laboratory of Fire Science of Chinese Academy of Sciences in 2007. His main research interests include smoke movement and ventilation control method in buildings, fire dynamics simulation and performance based fire safety design.

Rui TU. He is a Graduate of Zhengzhou University from September 2010 to present. He received his Bachelor's degree in Safety Science and Engineering from Chemical Engineering and Energy of Zhengzhou University in 2010. His research interest includes smoke spread and control method in tunnels.

Jianpeng YANG. He is a Senior Engineer of Nanyang Fire Detachment of Fire Bureau of Henan province. He received his PhD degree in Safety Science and Engineering in 2008. His research interest includes fire safety management in buildings and performance based fire safety design.

Tian Shui LIANG. He is a Lecturer of School of Chemical Engineering and Energy of Zhengzhou University. He received his $\mathrm{PhD}$ degree from Department of Civil and Architectural Engineering of City University of Hong Kong in 2012. His main research interests include physical and chemical processes in flame extinction, smoke movement and ventilation control method in buildings, and fire prevention technologies. 